02,05

\title{
Режимы взаимодействия намагниченных стопок ВТСП-лент
}

\author{
() А.И. Подливаев ${ }^{1,2}$, И.А. Руднев ${ }^{1}$ \\ ${ }^{1}$ Национальный исследовательский ядерный университет „МИФИ“, \\ Москва, Россия \\ ${ }^{2}$ Научно-исследовательский институт проблем развития научно-образовательного потенциала молодежи, \\ Москва, Россия \\ E-mail: AIPodlivayev@mephi.ru
}

Поступила в Редакцию 16 сентября 2021 г.

В окончательной редакции 16 сентября 2021 г.

Принята к публикации 11 октября 2021 г.

На основе модели критического состояния рассчитана сила взаимодействия пары магнитных линеек, представляющих собой наборы намагниченных стопок ВТСП-лент второго поколения. Рассмотрены режимы намагничивания взаимодействующих линеек внешним магнитным полем и характер перемагничивания стопок лент при многократных циклах сближения - удаления линеек друг от друга. Определена сила взаимодействия линеек в зависимости от расстояния между ними и от номера цикла.

Ключевые слова: высокотемпературные сверхпроводники, ВТСП-ленты второго поколения, магнитная левитация, сила взаимодействия, критическое состояние.

DOI: 10.21883/FTT.2022.02.51946.205

\section{1. Введение}

Источники неоднородного по пространству магнитного поля постоянно совершенствуются, что связано с их разнообразными техническими приложениями (см., например, обзор работы [1] и литературу в нем, а также работы [2-8]). Комбинация магнитов, создающих неоднородное магнитное поле [2-8] является одним из базовых элементов различных бесконтактных устройств, функционирующих на основе магнитной левитации (транспорт, бесконтактные подшипники и т. п.). Для создания магнитолевитационных систем необходимы источники магнитного поля, обладающие как высоким значением магнитной индукции, так и значительным градиентом магнитного поля. Градиентная конфигурация магнитного поля часто создается набором разнополярных постоянных магнитов. Одним из ограничений такого подходы является величина остаточной магнитной индукции современных постоянных магнитов, которая для распространённых магнитов на основе $\mathrm{NdFeB}$ находится в пределах 0.5 T. Повысить величину постоянного поля при сохранении высокого значения градиента можно за счет использования магнитов захваченного потока на основе сверхпроводников. Будучи намагниченными в сильном магнитом поле, такие структуры способны захватывать и сохранять огромные магнитные поля. Так, на объемных ВТСП-значения захваченного магнитного поля при низких температурах составляют $17.6 \mathrm{~T}[9,10]$ а на стопках ВТСП-лент - $17.7 \mathrm{~T}$ [11]. Остаточная намагниченность ВТСП связана с явлением пиннинга. Намагниченные ВТСП захватывают магнитный поток в виде вихрей Абрикосова, и могут долгое время сохранять на своей поверхности магнитную индукцию, превосходящую по величине индукцию постоянных маг- нитов [9-11]. Преимущество источника поля на основе ВТСП, обусловленное высокой напряженностью генерируемого магнитного поля, сопровождается рядом недостатков. Так при недостаточном охлаждении в намагниченном сверхпроводнике возможно возникновение термодинамической нестабильности $[12,13]$, которая может приводить к потере остаточного магнитного момента. Помимо этого, в системах, основанных на остаточной намагниченности ВТСП возможна деградация этой намагниченности как по прошествии некоторого времени (за счет крипа магнитного потока) [14], так и под действием внешних переменных магнитных полей [15]. Тем не менее, использование сверхпроводящих магнитов безусловно несет значительные преимущества за счет многократного увеличения амплитуды поля. В работе [16] проводится сравнительный анализ эффективности левитационных систем, в которых неоднородное магнитное поле создается как постоянными магнитами на основе $\mathrm{NdFeB}$, так и на основе пакетов ВТСПлент $\mathrm{GdBaCuO}$. В этой работе показано, что подъемная сила левитационных систем с источником градиентного магнитного поля выше для сверхпроводящих систем в случае, когда характерный размер неоднородности поля превышает величину $38 \mathrm{~mm}$ при температуре жидкого азота (или $6 \mathrm{~mm}$ при температуре жидкого неона), а при меньших размерах более эффективны системы на основе постоянных магнитов. В настоящей работе также изучается схема, в которой постоянный магнит отсутствует, а его роль играет намагниченная стопка ВТСП лент. Вместе с тем, эффекты перемагничивания сверхпроводников, могут вносить коррективы для дальнейшего использования магнитов захваченного потока в реальных левитационных системах. В работе [16] не исследовались процессы перемагничивания источ- 
ника градиентного магнитного поля (это приближение справедливо при сравнительно слабом взаимодействии частей левитационной системы). В данной статье впервые рассмотрен общий случай - исследованы процессы перемагничивания сверхпроводящих частей магнитолевитационной подвески под действием циклической нагрузки.

В настоящей работе мы изучаем неоднородные конфигурации токов ВТСП-лент, из которых состоит левитационная система. Неоднородные состояния магнитных пленок различного типа изучаются в рамках различных подходов в соответствие с масштабом неоднородности. На атомном уровне могут применяться расчеты в рамках теории функционала плотности [17]. Неоднородность обусловленная микроскопической зернистой структурой может приводить к необычным физическим явлениям, например, появлению фазовых переходов по току, аналогичных наблюдаемым в гранулированных сверхпроводниках $\mathrm{YBa}_{2} \mathrm{Cu}_{3} \mathrm{O}_{7-x}[18]$. В работах $[19,20]$ экспериментально показано влияние грануляции структуры ВТСП на гистерезисный характер магнитосопротивления. Для левитационных приложений характерен интервал размеров неоднородностей, превышающий несколько миллиметров. Именно такой характерный размер неоднородностей магнитного поля и токов будет рассмотрен в настоящей статье.

\section{2. Постановка задачи}

Рассмотрим систему, состоящую из двух параллельных наборов ВТСП-лент - магнитной подвески. Проекция ВТСП-подвески на плоскость $(X, Z)$ представлена на рис. 1. Сверхпроводящая часть подвески состоит из нижней неподвижной части, и верхней части, которая перемещается в вертикальном направлении с координатой $Z(t), t-$ время. В начальный момент времени $t=0$ обе части подвески не намагничены. Намагничивание обеих частей производится внешним магнитным полем $\mathbf{B}_{\text {ext }}(t)$, однородным по пространству и переменным по времени. Единственная ненулевая нормальная компонента этого поля $B_{Z}(t)=B_{\max } t$ при $0 \leq t \leq t_{0}, B_{Z}(t)=B_{\max }(2-t)$ при $t_{0} \leq t \leq 2 t_{0}$ и $B_{Z}(t)=0$ при $2 t_{0} \leq t$. Также как в работах $[16,21,22]$ в качестве сверхпроводящей основы была выбрана ВТСП-лента со сверхпроводником $\mathrm{GdBaCuO}$ на подложке из сплава хастеллой и защитными слоями из меди и серебра. Полная толщина ВТСП-ленты равна $0.1 \mathrm{~mm}$, а толщина сверхпроводящего слоя $d=1.5 \mu \mathrm{m}$. Ширина ВТСП-ленты $a=12 \mathrm{~mm}$. Намагничивание проводится адиабатически медленно, то есть величина характерного время намагничивания $t_{0}$ выбирается так, чтобы остаточные наведенные в сверхпроводящих стопках токи не зависели от этой величины. Амплитуда $B_{\max }$, необходимая для предельного намагничивания образца зависела от количества отдельных лент $N_{l}$ в стопке. При расчетах нами выбраны две величины $N_{l}=1$ и 20 , и соответствующие им значения

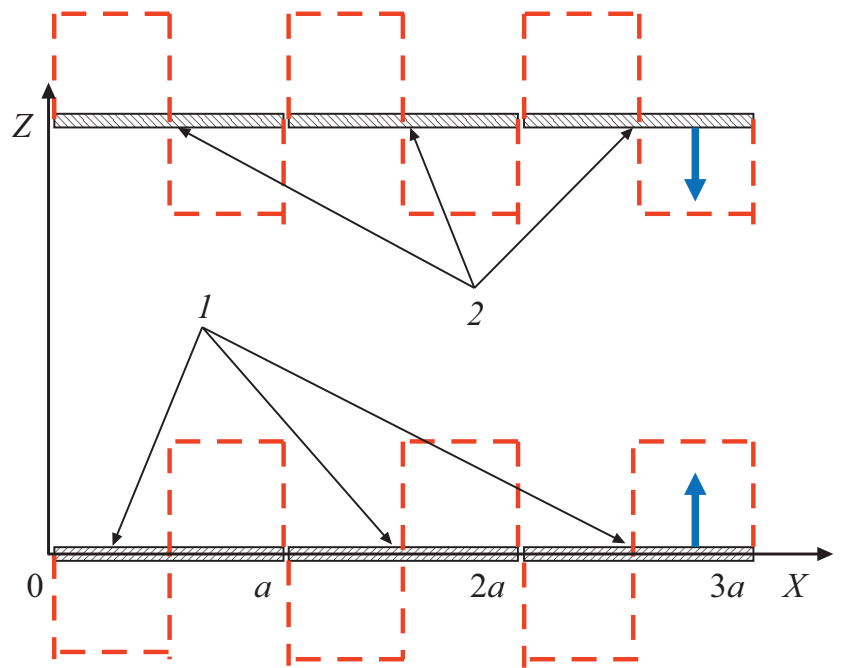

Рис. 1. Структура магнитной подвески. 1 и $2-$ стопки ВТСП-лент нижней и верхней частей подвески соответственно. Каждая стопка изолирована от соседней, имеет ширину $a$ и содержит $N_{l}$ отдельных лент, размещенных одна над другой в направлении $Z$. В направлении оси $Y$ длина стопки полагается неограниченной. В направлении оси $X$ структура полагается периодической (величина периода равна $a$ ). Штриховой линией схематически изображена плотность тока $j_{Y}$, наведенная в верхней и нижней частях подвески внешним магнитным полем. Жирными стрелками справа обозначены направления намагниченности стопок нижней и верхней частей подвески.

$B_{\max }=1$ и 5 T. Выбор максимальной величины $N_{l}=20$ сделан на основании данных работы [16] в которой было показано, что толщина пакетов ВТСП-лент для левитационных приложений должна быть ограничена. Действительно, из данных этой работы следует, что магнитное поле над периодической ВТСП-линейкой с толщиной, равной половине периода $(6 \mathrm{~mm}$ для лент шириной $12 \mathrm{~mm}$ ) практически не отличается от магнитного поля ВТСП-стопки бесконечной толщины. Исходя из этого, в настоящей работе мы ограничились сравнением стопок, состоящих из 1 и 20 лент (толщины пакетов 0.1 и $2 \mathrm{~mm}$ ), полагая дальнейшее увеличение высоты стопок неэффективным. Временная зависимость расстояния $Z(t)$ между верхней и нижней частями подвески выбрана в следующем виде: $Z(t)=a$ при $0 \leq t \leq 2 t_{0}$ и $Z(t)=a\left[1+q+(1-q) \cos \left(2 \pi\left(t / t_{0}-2\right)\right)\right] / 2$ при $2 t_{0} \leq t$. Такая форма зависимости $Z(t)$ представляется наиболее простой и адекватной при одновременном описании и начального намагничивания, и последующих осцилляций. Параметр $q$ определяет амплитуду колебаний и задает минимальное расстояние, на которые сближаются слои. На первом временном участке $t \leq 2 t_{0}$ межплоскостное расстояние не изменяется - это этап намагничивания. На нем межплоскостное расстояние фиксировано, и внешнее поле не равно нулю, а после него - поле равно нулю, а расстояние осциллирует. В последующих расчетах величины $q=0.1,0.05$ и 0.02 . 

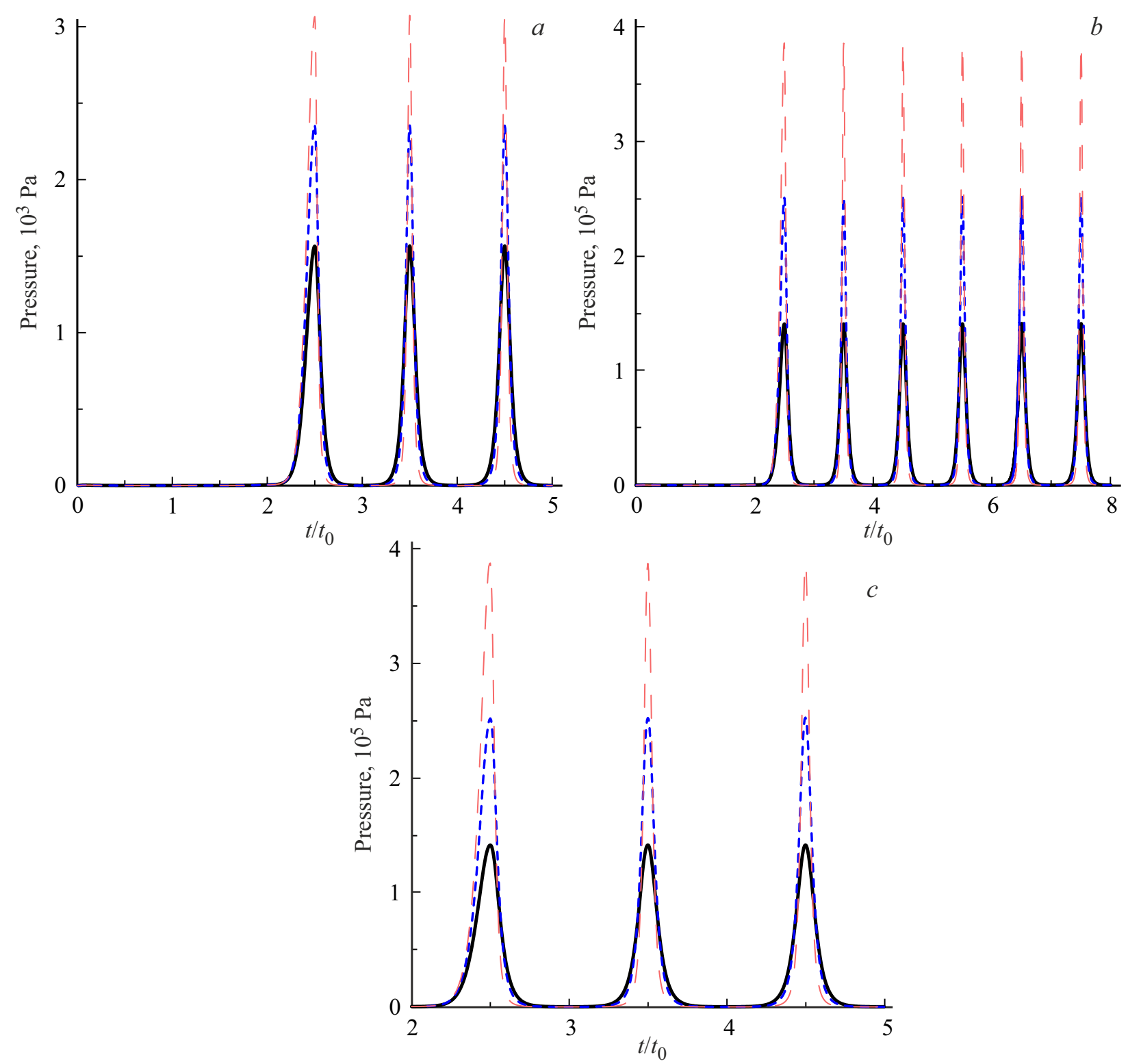

Рис. 2. Временная зависимость величины давления верхней части магнитной подвески на нижнюю. Этап намагничивания и первые 3 периода приближения верхней части к нижней $N_{l}=1(a)$. Этап намагничивания и 6 периодов приближения верхней части к нижней $N_{l}=20(b)$. Первые 3 периода $N_{l}=20(c)$. Жирная сплошная линия $-q=0.1$, средняя штриховая линия $q=0.05$, тонкая штриховаяя линия $-q=0.02$.

Осцилляторный характер изменения расстояния между верхней и нижней частями подвески позволяет изучить переходные процессы работы подвески при переменных вертикальных нагрузках и определить установившийся режим при таких колебаниях высоты $Z(t)$.

Законы индукции, Био-Савара [23] и закон Ома, определяющие сверхпроводящие токи имеют следующий вид:

$$
\begin{gathered}
\frac{\partial \mathbf{B}}{\partial t}=-\nabla \times \mathbf{E}, \\
\mathbf{B}(\mathbf{R})=\mathbf{B}_{\mathrm{ext}}(t)+\frac{\mu_{0}}{4 \pi} \int_{S} \frac{\mathbf{j}(\mathbf{r}) \times(\mathbf{R}-\mathbf{r})}{|\mathbf{R}-\mathbf{r}|^{3}} d^{3} r, \mathbf{E}=\rho(|\mathbf{j}|) \mathbf{j} .
\end{gathered}
$$

Символами $\mathbf{B}, \mathbf{E}$ и $\mathbf{j}$ обозначены вектора магнитной индукции, напряженности электрического поля и плотности наведенных сверхпроводящих токов соответственно. $S$ - область сверхпроводящих токов, $\mu_{0}$ и $\rho(\mathbf{j} \mid)-$ магнитная постоянная и удельное сопротивление сверхпроводника, определяемое аналогично работам $[16,22]$ следующим образом:

$$
\begin{gathered}
\rho(\mathbf{j}, B)= \begin{cases}0, & |\mathbf{j}|<j_{c}(B), \\
\rho_{0}\left[|\mathbf{j}|-j_{c}(B)\right]^{2}, & j_{c}(B) \leq|\mathbf{j}|,\end{cases} \\
j_{c}(B)=A_{1} \exp \left(-|B| / \beta_{1}\right)+A_{2} \exp \left(-|B| / \beta_{2}\right) .
\end{gathered}
$$



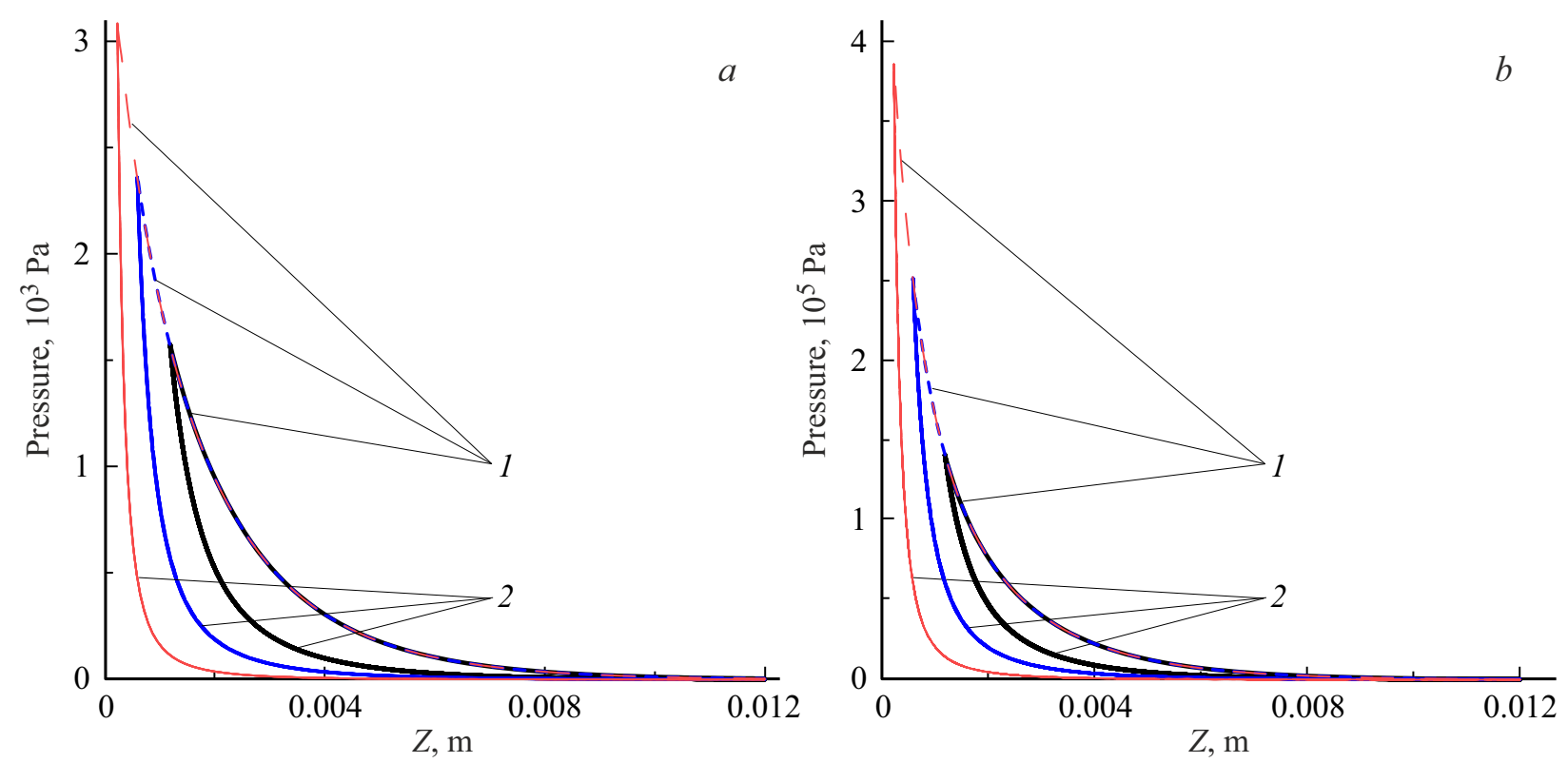

Рис. 3. Зависимость величины давления верхней части магнитной подвески на нижнюю от расстояния между ними. Число лент в стопке $N_{l}=1(a), N_{l}=20(b)$. Представлены 6 периодов приближения верхней части к нижней. Жирная сплошная линия $q=0.1$, средняя штриховая линия $-q=0.05$, тонкая штриховая линия $-q=0.02 .1-$ участки зависимости, соответствующие временному интервалу $2 \leq t / t_{0} \leq 2.5$ (этап первого сближения частей подвески); 2 - участки зависимости, соответствующие временному интервалу $2.5 \leq t / t_{0} \leq 8$ (режим установившихся осцилляций).

Для объемной критической плотности сверхпроводящего тока в $\mathrm{GdBaCuO}$ при температуре жидкого азота $A_{1}=2.3665 \cdot 10^{8} \mathrm{~A} / \mathrm{m}^{2}, A_{2}=1.7884 \cdot 10^{8} \mathrm{~A} / \mathrm{m}^{2}$, $\beta_{1}=0.1175 \mathrm{~T}$ и $\beta_{2}=1.2238 \mathrm{~T}$ [18]. Детальное описание уравнений (1),(2), а также алгоритм численного решения данной задачи представлены в работе [24], и ввиду громоздкости, в данной статье не приводятся. Двумерная плотность сверхпроводящих токов $J$, имеющая размерность $\mathrm{A} / \mathrm{m}$ связана с объемной плотностью $j$ соотношением $J=j d$. Сила действия индукции магнитного поля нижней части подвески на сверхпроводящие токи верхней части определялась законом Ампера.

\section{3. Результаты}

На рис. 2 представлена временная зависимость силы взаимодействия двух частей подвески, отнесенная к единице поверхности для подвески, состоящей из пакетов ВТСП-лент при $N_{l}=1$ и 20. При выбранной зависимости $Z(t)$ части подвески удалены друг от друга на максимальное расстояние, равное $12 \mathrm{~mm}$. Величина $Z(t)$ максимальна при $0 \leq t / t_{0} \leq 2$ и при $t / t_{0}=3,4,5, \ldots$. На рис. 2 видно, что при максимальном удалении магнитное взаимодействие практически отсутствует. Сила отталкивания частей максимальна при минимальном расстоянии $Z(t)$, которое достигается в моменты времени $t / t_{0}=2.5,3.5,4.5,5.5, \ldots$ На крупномасштабном рис. 2,c при $N_{l}=20$ видно, что форма первого пика для всех значений параметра $q$ отличается от пиков последующих, совпадающих между собой. На рис. 3 представлена временная зависимость силы взаимодействия двух частей подвески от расстояния между ними на временном интервале $2 \leq t / t_{0} \leq 8$. Видно, что форма кривых на временном интервале $2 \leq t / t_{0} \leq 2.5$ (на рис. 3 участки отмечены цифрой 1 ) в подвесках каждого типа отличаются от наблюдаемых на интервале $2.5 \leq t / t_{0} \leq 8$ (отмечены цифрой 2), где движение строго периодично. Траектория периодического движения одномерного тела в осях сила-смещение при наличии трения имеет вид замкнутой гистерезисной кривой. Площадь под этой кривой равна энергии, рассеянной за счет сил трения в течение одного цикла. Исходя из формы кривых на рис. 3 , диссипация энергии наблюдается только на участке $2 \leq t / t_{0} \leq 2.5$. Это рассеяние обусловлено выделением энергии, связанным с отрывом системы вихрей Абрикосова от центров пиннинга ВТСП. На интервале $2.5 \leq t / t_{0} \leq 8$ гистерезисная петля не наблюдается, т.е. диссипация энергии отсутствует и магнитная подвеска является идеальной нелинейной пружиной. Жесткость этой пружины зависит от величины амплитуды осцилляций и существенно возрастает по мере сближения частей подвески. Взаимное давление частей подвески в этом случае также возрастает и может достигать величины $3100 \mathrm{~Pa}$ для однослойной подвески, а пиковое давление отталкивания в многослойной подвеске $\left(N_{l}=20\right)$ равно $400000 \mathrm{~Pa}(\sim 4$ атмосферы $)$. Если бы отсутствовало подавление критического тока магнитным полем (смотри выражение (2)), то зависимость пикового давления отталкивания была бы строго квадратичной, поскольку эта величина зависит от произведения плот- 

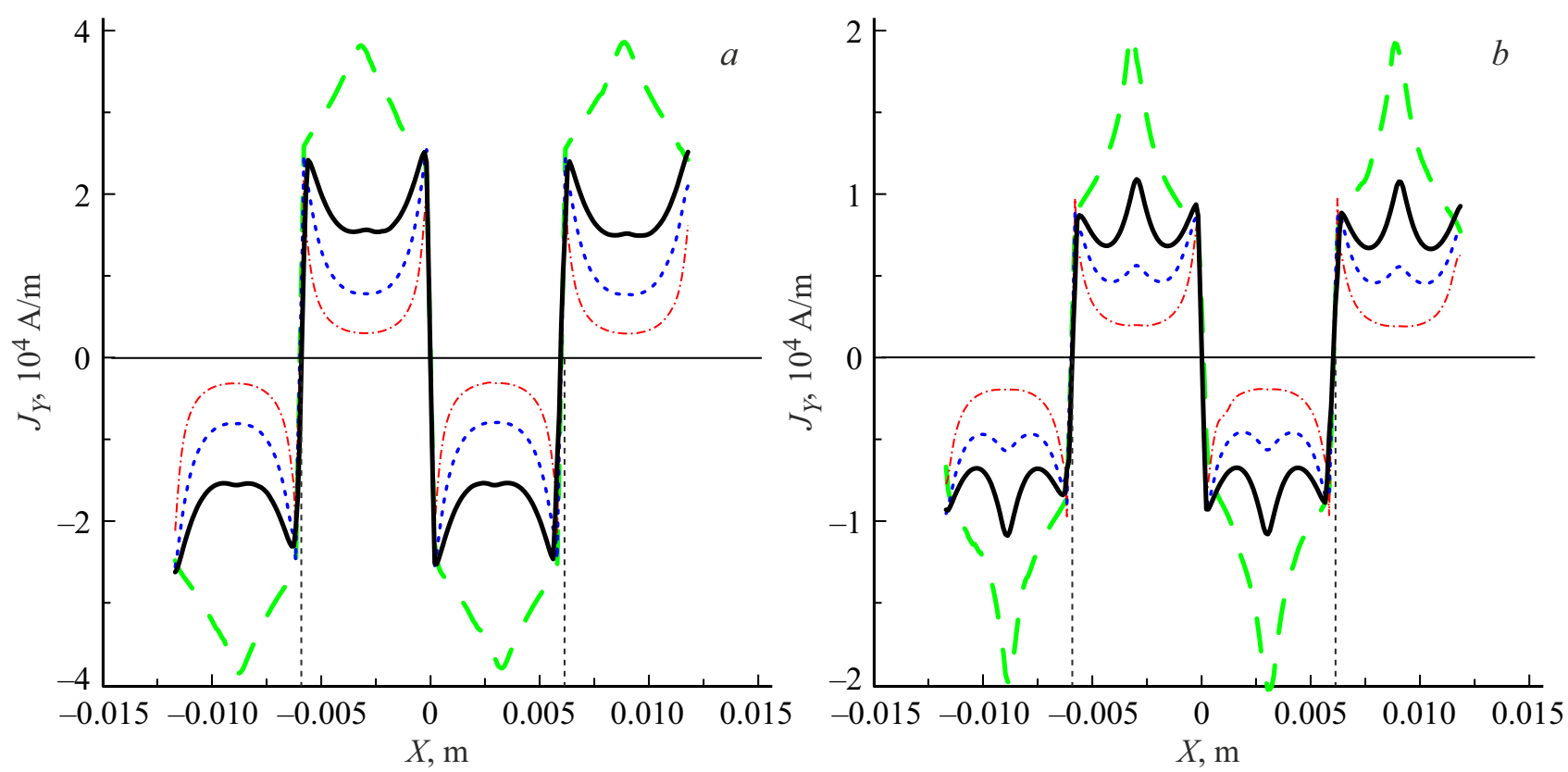

Рис. 4. Двумерная плотность тока $J_{Y}(X)(\mathrm{A} / \mathrm{m})$ в нижней части подвески, состоящей из одной $\left(N_{l}=1\right)$ ленты $(a)$ и средняя двумерная плотность тока $J_{Y}(X)$, приходящаяся на одну ленту в стопке из 20 лент $\left(N_{l}=20\right)(b)$. Жирная штриховая линия плотность после намагничивания до первого сближения частей подвески $\left(t / t_{0}=2\right)$. Жирная сплошная линия $-q=0.1$, пунктирная линия $-q=0.05$, штрих-пунктирная линия $-q=0.02$ в конечный момент времени $t / t_{0}=8$ после 6 осцилляций. Вертикальными тонкими штриховыми линиями отмечены границы пакетов лент.

ности токов одной части подвески на нормальную компоненту магнитного поля, создаваемого второй частью. В этом случае сила в многослойной подвеске превосходила бы соответствующую величину в однослойной в $\left(N_{l}\right)^{2}=400$ раз, в то время как подавление критического тока магнитным полем уменьшает это отношение до величины $400000 / 3100=129$ раз. Взаимное влияние токов двух частей подвески через магнитное поле иллюстрирует рис. 4, на котором представлены распределения двумерной плотности сверхпроводящих токов $J_{Y}(X)$ после намагничивания, но до изменения расстояния между частями подвески и после 6 циклов сближения частей подвески. Сопоставление данных рис. 4, $a, b$ показывает, что плотность токов в каждой отдельной ленте многослойной стопки $\left(N_{l}=20\right)$ заметно ниже аналогичной величины в однослойной стопке (максимальное значение плотности тока ожидаемо достигается при $X= \pm a / 2$, и равно $\sim 40000 \mathrm{~F} / \mathrm{m}$ в однослойной подвеске, в то время как в многослойной достигает величины $\sim 20000 \mathrm{~A} / \mathrm{m})$. Процесс размагничивания, в целом, проходит интенсивнее в однослойной стопке. Об интенсивности можно судить, например, по тому, что при параметре $q=0.1$ в многослойной стопке после осцилляций хорошо сохраняется центральный пик плотности тока (при $X= \pm a / 2$, рис. $4, b)$, в то время как в однослойной стопке он почти исчезает. При этом наибольшее падение плотности критического тока до начала осцилляций наблюдается вблизи краев ленты, где собственное магнитное поле пакета максимально - смотри рис. 5, на котором пред-

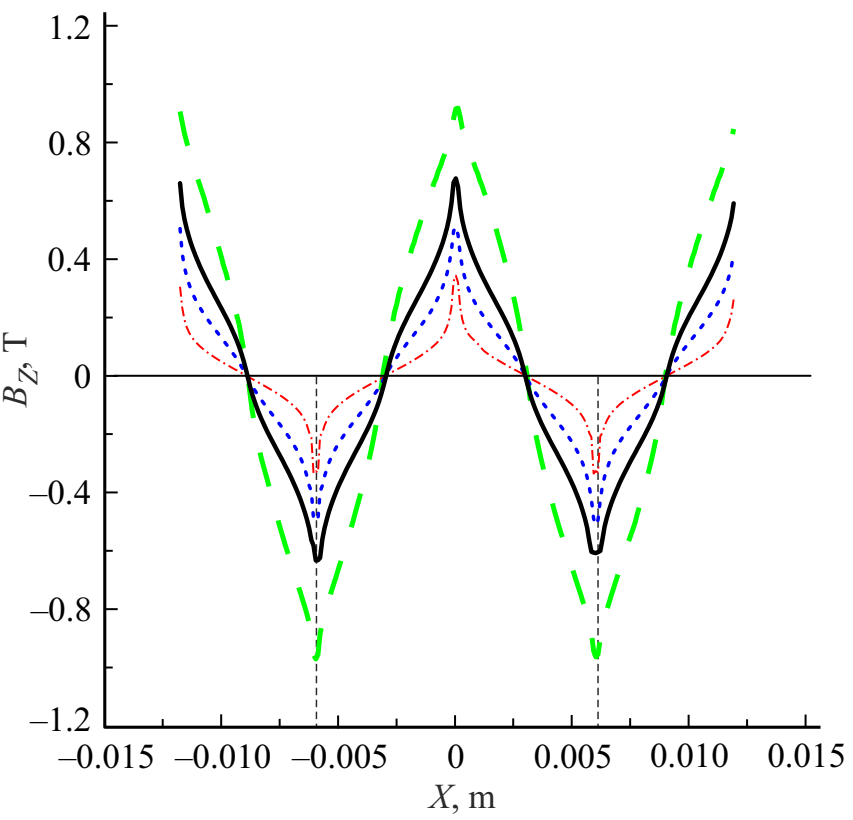

Рис. 5. Величина нормальной компоненты индукции магнитного поля над поверхностью подвески при максимальном удалении ее частей друг от друга. Жирная штриховая линия распределение после намагничивания до первого сближения частей подвески $\left(t / t_{0}=2\right)$. Жирная сплошная линия $q=0.1$, пунктирная линия $-q=0.05$, штрих-пунктирная линия $-q=0.02$ в конечный момент времени $t / t_{0}=8$ после 6 осцилляций. 
ставлена зависимость нормальной компоненты индукции магнитного поля $B_{Z}(X)$ на поверхности многослойной подвески при максимальном удалении верхней части подвески от нижней. На этом рисунке также видно, что даже при максимальном размагничивании $(q=0.02$, см. штрих-пунктирную линию) пиковые значения индукции магнитного поля существенно превышают значение величины $\beta_{1}=0.1175 \mathrm{~T}$, которая определяет подавление плотности критического тока магнитным полем в двухэкспоненциальном приближении (2), что и объясняет сильную неоднородность плотности сверхпроводящего тока в обеих частях подвески.

\section{4. Заключение}

В настоящей работе представлена принципиально новая схема левитационной подвески на основе пакетов сверхпроводящих лент без применения традиционных ферромагнитных материалов. Теоретический расчет показал существование практически значимых консервативных режимов работы подвески, при которых в предложенной системе отсутствует диссипация энергии (неизбежно сопровождаемая нагревом сверхпроводников). Показано, что при многократных осцилляциях, установление консервативного режима происходит в течении одной (первой) осцилляции. Показано, что подъемная сила подвески возрастает в $~ 130$ раз при увеличении числа лент в сверхпроводящих частях подвески от 1 до 20, но дальнейшее увеличение числа лент не изменяет существенно подъемную силу вследствие подавления сверхпроводящего тока в частях подвески собственным магнитным полем. Полученные в работе теоретические данные обладают предсказательной силой распределение магнитного поля на поверхности частей подвески может быть экспериментально измерено методами холловской магнитометрии. Также представляется интересным определить изменение левитационной силы и рассеиваемую мощность при поперечном смещении частей подвески относительно друг друга.

\section{Финансирование работы}

Работа выполнена при финансовой поддержке Российского научного фонда (проект 17-19-01527).

\section{Конфликт интересов}

Авторы заявляют, что у них нет конфликта интересов.

\section{Список литературы}

[1] F. Balci, A. Bingolbali, N. Dogan, M. Irfan. Письма в ЖТФ 47, 4, 3 (2021).

[2] I. Valiente-Blanco, E. Diez-Jimenez, C. Cristache, M.A. Alvarez-Valenzuela, J.L. Perez-Diaz. Tribology Lett. 6 (2013). DOI: $10.1007 / \mathrm{s} 11249-013-0204-0$
[3] F. Antoncik, M. Lojka, T. Hlasek, V. Bartunek, I. ValienteBlanco, J.L. Perez-Diaz, O. Jankovsky. Supercond. Sci. Technol. 33, 045010 (2020). https://doi.org/10.1088/1361-6668/ab6ebe

[4] M. Osipov, I. Anishenko, A. Starikovskii, D. Abin, S. Pokrovskii, A. Podlivaev, I. Rudnev. Supercond. Sci. Technol. 34, 035033 (2021).

DOI.org/10.1088/1361-6668/abda5a

[5] J.G. Storey, M. Szmigie, F. Robinson, S.C. Wimbush, R.A. Badcock. IEEE Transact. Appl. Supercond. 30, 4, 600706 (2020). DOI: 10.1109/TASC.2020.2982884.

[6] Y. Miyazaki, K. Mizuno, T. Yamashita, M. Ogata, H. Hasegawa, K. Nagashima, S. Mukoyama, T. Matsuoka, K. Nakao, S. Horiuch, T. Maeda, H. Shimizu. Cryogenics 80, 234 (2016).

[7] P. Bernstein, J. Noudem. Supercond. Sci. Technol, 33, 3, 033001 (2020). DOI: 10.1088/1361-6668/ab63bd

[8] Kun Liu, Wenjiao Yang, Guangtong Ma, Loïc Quéval, Tianyong Gong, Changqing Ye, Xiang Li, Zhen Luo. Supercond. Sci. Technol. 31, 015013 (2018). doi.org/10.1088/1361-6668/aa987b

[9] M. Tomita, M. Murakami. Nature 421, 517 (2003).

[10] J.H. Durrell, A.R. Dennis, J. Jaroszynsk, M.D. Ainslie, K.G.B. Palmer, Y-H. Shi, A.M. Campbell, J. Hull, M. Strasik, E.E. Hellstrom. Supercond. Sci. Technol. 27, 8, 082001 (2014).

[11] A. Patel, A. Baskys, T. Mitchell-Williams, A. McCaul, W. Coniglio, J. Hänisch, B.A. Glowacki. Supercond. Sci. Technol. 31, 09LT01 (2018).

[12] В.Р. Романовский. ЖТФ 87, 1, 49 (2017). DOI: $10.21883 /$ JTF.2017.01.44018.1823

[13] В.Р. Романовский. ЖТФ 87, 4, 540 (2017). DOI: 10.21883/JTF.2017.04.44313.1943x90

[14] D. Abin, M. Osipov, S. Pokrovskii, I. Rudnev. IEEE Trans. Appl. Supercond. 26, 3, 8800504 (2016). DOI: 10.1109/TASC.2016.2525924.

[15] S. Pokrovskii, A. Dmitry, M. Osipov, I. Rudnev. IEEE Trans. Appl. Supercond. 26, 3, 8201304 (2016). DOI: 10.1109/TASC.2016.2533573.

[16] А.И. Подливаев, И.А. Руднев. ФТТ 63, 10, 1514 (2021).

[17] T.P. Yadav, A. Srivastava, G.C. Kaphle. ФTT 63, 2, 249 (2021).

[18] В.В. Деревянко, Т.В. Сухарева, В.А. Финкель. ФТТ 60, 3, 465 (2018).

[19] С.В. Семенов, Д.А. Балаев, М.И. Петров. ФТТ 63, 7, 854 (2021).

[20] С.В. Семенов, Д.А. Балаев. ФТТ 62, 7, 1008 (2020).

[21] С.В. Самойленков, В.И. Щербаков, Д.Р. Кумаров, Д.А. Горбунова. Письма в ЖТФ 46, 1, 28 (2020). DOI: 10.21883/PJTF.2020.01.48860.18047

[22] А.И. Подливаев, И.А. Руднев. ФТТ 63, 6, 712 (2021).

[23] Л.Д. Ландау, Е.М. Лифшиц. Теория поля. Наука, М. (1988). $512 \mathrm{c}$.

[24] A.I. Podlivaev, I.A. Rudnev. Supercond. Sci. Technol. 30, 035021 (2017). DOI: 10.1088/1361-6668/aa55aa

Редактор К.В. Емцев 